\title{
Multidisciplinary Teams in the Management of Placenta Accreta Spectrum Disorders
}

\author{
Brett D. Einerson $^{1} \cdot$ Robert M. Silver ${ }^{1}$ \\ Published online: 11 July 2019 \\ (C) Springer Science+Business Media, LLC, part of Springer Nature 2019
}

\begin{abstract}
Purpose of Review The three-fold purpose of this review is to summarize recent evidence supporting the use of a multidisciplinary team (MDT) approach in patients with placenta accreta spectrum (PAS), to describe essential members and processes of the PAS MDT, and to highlight necessary resources for both planned and unexpected cases of PAS.

Recent Findings Maternal outcomes in women with PAS are improved when an antenatal diagnosis occurs and referral is made to centers with MDT specialization and experience in treating PAS. New guidelines unequivocally recommend referral to highervolume, experienced PAS centers of excellence.

Summary The diagnosis and treatment of patients with PAS are complex, resource-intensive, and pose high risk of maternal morbidity and mortality. Recent evidence suggests that stable, experienced MDTs focused on continuous systems improvement at high-volume tertiary care centers are superior to standard obstetric management for patients with PAS. Referral to a center of excellence is indicated when PAS is suspected.
\end{abstract}

Keywords Cesarean hysterectomy · Multidisciplinary team · Centers of excellence $\cdot$ Patient safety $\cdot$ Levels of maternal care · Transfusion · Obstetric anesthesiology

\section{Introduction}

Few health conditions are as complex, resource-intensive, and potentially deadly as placenta accreta spectrum disorders (PAS). Detailed descriptions of the etiology, pathophysiology, and epidemiology of PAS are discussed elsewhere in this edition.

PAS is now the most common reason for peripartum hysterectomy in the USA [1•] and an important contributor to maternal death. Surgical management of PAS can result in abrupt, unexpected, life-threatening hemorrhage. Dilutional coagulopathy, transfusion-related complications, cardiorespiratory overload, electrolyte abnormalities, acute kidney injury, and intensive care unit admission are very real possibilities in the setting of PAS. Surgery for PAS is frequently complicated by injury to the bladder, bowel, or ureters. Also,

This article is part of the Topical Collection on Aberrant Placentation: Contemporary Management of Placenta Accreta

Brett D. Einerson

Brett.Einerson@hsc.utah.edu

1 University of Utah Health, Salt Lake City, UT, USA
PAS often goes undetected until the time of delivery resulting in emergent surgery and care in settings not suited for massive hemorrhage.

The purposes of this review are (1) to review recent evidence supporting the use of a multidisciplinary team (MDT) approach, (2) to describe essential members and processes of the multidisciplinary PAS team, and (3) to describe resources needed for both planned and unexpected cases of PAS.

\section{Multidisciplinary Teams Improve Outcomes}

Maternal outcomes are improved when patients with PAS are managed by multidisciplinary teams (MDTs) in experienced referral centers. In a population-based study of all PAS cases in Utah from 1996 to 2008, Eller and colleagues demonstrated improved maternal outcomes when cases of PAS were managed in hospitals with a MDT care model [2•]. They compared the outcomes of 141 cases managed at two tertiary care PAS referral centers (MDT care) to those at 26 other hospitals in Utah that did not meet criteria for MDT care (standard obstetric care). Women with PAS managed by multidisciplinary care had a significantly lower frequency of a composite early 
maternal morbidity outcome including prolonged admission to the intensive care unit, large-volume blood transfusion, coagulopathy, ureteral injury, or early reoperation compared to women managed by standard obstetric care (47\% vs $74 \%, p=$ 0.026). After controlling for clinical factors, the odds of early major maternal morbidity with MDT care was about one-fifth that with standard obstetric care (aOR 0.22 [95\% confidence interval, 0.07-0.71]).

Even at tertiary care referral centers, care of women with PAS can be improved by implementing a MDT care approach. Shamshirsaz and colleagues showed that a change from standard care of patients with PAS to coordinated multidisciplinary care improves maternal outcomes [3]. In this study, the authors evaluated the surgical outcomes of 90 patients with PAS managed before and after a system-wide practice change at three referral centers. Before the change to MDT care, patients were managed by individual clinicians without a standardized protocol. After the intervention, a comprehensive MDT approach was utilized. Women with PAS who were cared for by MDTs had lower median blood loss (2.1 1 versus $31, P=0.025$ ) and were less likely to require emergent surgery (23\% vs $64 \% ; P=0.001)$. These outcomes were achieved despite there being a higher frequency of percreta in the MDT study group (after the intervention) $(P=0.008)$.

Experience and stability of the MDT also matter. In a single-site study, Shamshirsaz and colleagues evaluated outcomes of patients with PAS over time after establishing a MDT care model [4]. Three maternal-fetal medicine specialists served as central leaders for the accreta team over the study period from 2011 until 2016, managing a total of 118 total cases. The authors compared two time periods, before and after April 2014. Median blood loss, red cell transfusion, and crystalloid transfusion were less common in the latter group, suggesting that team learning and experience improved patient safety outcomes over time. Impressively, the need for massive transfusion was one fifth as frequent in the latter time period $(5.1 \%$ vs $25.4 \%, P<0.01)$.

The benefit of delivery at a referral center extends beyond cases of PAS to all women with massive obstetric hemorrhage requiring peripartum hysterectomy. In a study of 2209 women undergoing peripartum hysterectomy in a nationwide US database of over 500 hospitals, Wright and colleagues [1•] found that women undergoing peripartum hysterectomy for obstetric hemorrhage, whether or not PAS is present, are less likely to die at high volume centers (aOR 0.29 [95\% CI 0.10-0.88]). They also showed lower odds of perioperative surgical complications (aOR 0.66 [95\% CI 0.477-0.93]) and intensive care unit utilization (aOR 0.53 [95\% CI 0.34-0.83]) at high volume centers. PAS was the most common reason for peripartum hysterectomy, accounting for $35 \%$ of cases.

The central role of experienced MDTs in the management of PAS is reinforced by recent international guidelines. The American College of Obstetricians and Gynecologists
(ACOG) Obstetric Care Consensus [5] states that the "optimal management [of PAS] involves a standardized approach with comprehensive multidisciplinary care team accustomed to management of placenta accreta spectrum." ACOG has also supported regionalization of care for women with antenatally diagnosed PAS, who should, along with all women at "extreme risk of massive hemorrhage at delivery," be referred to and managed in at least a level III hospital according to the levels of maternal care paradigm [6]. The Royal College of Obstetricians and Gynecologists (RCOG) from the United Kingdom Green Top Guideline [7] states that "women diagnosed with placenta accreta spectrum should be cared for by a multidisciplinary team in a specialist cent[er] with expertise in diagnosing and managing invasive placentation."

Despite data demonstrating that delivery at a hospital with a multidisciplinary PAS team is beneficial, and straightforward guidelines calling for referral to highly experienced maternity centers, many patients with PAS are managed outside these optimal settings. One important reason is that patients with PAS may not be referred. In a survey of the American College of Obstetricians and Gynecologists, less than onequarter $(23 \%)$ of general OBGYNs refer women with PAS to a sub-specialist [8]. Another important reason for delivery outside of MDT care settings is missed diagnosis. Large population-based studies show that only half of patients with PAS are diagnosed antenatally $[9,10]$. The barriers to diagnosis and referral are largely unstudied.

\section{The Importance of Diagnosis and Referral}

Management of PAS at a referral center is unlikely to occur if an antenatal diagnosis is missed. Making the diagnosis is critical and marks the first step in the MDT subspecialty care of patients with PAS. When antenatal diagnosis occurs, the risk of adverse maternal outcomes is significantly decreased [11, 12]. A detailed description of the imaging modalities utilized for diagnosis of PAS is discussed elsewhere in this edition.

The decision to refer to more specialized PAS centers depends on several factors. Among them are the skill and experience of radiologists and maternal-fetal medicine subspecialists to make a diagnosis of PAS, the experience and volume of surgical specialists and anesthesiologists for PAS and (most importantly) the blood bank resources available at the center. Indications for referral to placenta accreta spectrum Centers of Excellence (CoE) are listed in Table 1 [13•].

\section{Components of a Multidisciplinary Accreta Team}

Multidisciplinary collaboration and availability of expert subspecialists are keys to safe care for patients with 
Table 1 Consider referral to Centers of Excellence for diagnosis and management in patients with clinical or sonographic risk factors for placenta accreta spectrum

Clinical risk

1. Multiple prior cesarean sections

2. Placenta previa

3. History of endometrial ablation

4. Previous uterine surgery

5. First- or second-trimester bleeding with other risk factors for placenta accreta spectrum

Sonographic risk

1. Abnormal placental appearance

2. Abnormal uterine shape

3. Abnormal vascularity of myometrial wall

4. Cesarean scar pregnancy

Adapted from Silver. Placenta accreta: center of excellence. Am J Obstet Gynecol 2015 [13••]

PAS. Suggested members of a PAS MDT are shown in Table 2. Centers should be able to quickly mobilize team members $24 \mathrm{~h}$ per day, year-round.

A well-stocked blood bank is a non-negotiable component of a PAS CoE. Although the median blood loss at the time of PAS surgery is reported as 2-3 1, it is not uncommon for PAS cases to have blood loss of more than 10 1. Hemorrhage is often abrupt, rapid, and life-threatening. The blood bank should be able to deliver multiple blood products quickly, $24 \mathrm{~h}$ per day. It should have a defined massive transfusion protocol (MTP), and ample supply of packed red cells, fresh frozen plasma (FFP), cryoprecipitate, platelets, and fibrinogen. Transfusion medicine specialists, who can guide preparation and delivery of blood products in the case of massive hemorrhage, are indispensable members of the PAS team. Ideally, blood

\section{Table 2 Members of a PAS MDT}

1. Experienced obstetricians and maternal-fetal medicine specialists

2. Experienced diagnostic imaging experts

3. Transfusion medicine and blood bank specialists

4.Obstetric anesthesiologists, or anesthesiologists with experience in massive obstetric hemorrhage

5. Expert pelvic surgeons (may be, though not exclusively, gynecologic oncologists)

6. Interventional radiologists

7. Urologists

8. General surgeons, trauma surgeons, and/or vascular surgeons

9. Critical care experts (adult and neonatal)

10. Neonatologists

11. Expert placental pathologists

12. Psychological and social support staff

13. Research staff products should be available in the room prior to starting the accreta surgery case.

Equally important is the presence and active participation of obstetric anesthesiologists or anesthesiologists with advanced expertise in massive obstetric hemorrhage. These experts keep the patient alive through episodes of rapid exsanguination, hemodynamic instability, and potentially prolonged surgery. Obstetric anesthesiologists monitor the cardiorespiratory, hemodynamic, and hematologic status of the patient and have unique knowledge of the physiologic changes of pregnancy and postpartum. They administer vasopressors and blood products. When possible, preoperative consultation of PAS patients with expert anesthesiologists is preferred. Anesthesiologists also aid in the preand peri-operative management decisions and optimization of medical comorbidities and serve as key members of the pre-operative planning team.

An on-site interventional radiology service is also important. Although the use of routine or prophylactic interventional radiology procedures (e.g., intermittent endovascular aortic occlusion or pelvic artery balloon catheter occlusion) for PAS is controversial, it is to a center's great advantage to have the option of postoperative embolization for active bleeding. This service is also a routine part of the care for patients who opt for conservative management of PAS (discussed elsewhere). Therefore, an experienced interventional radiologist should be a member of the PAS team. Expertise in diagnostic imaging of PAS is also valuable to optimally plan the surgical approach (discussed in chapter X).

Intensive care specialists are also key members of the PAS team. Up to half of all patients undergoing cesarean hysterectomy require ICU admission $[3,11,12]$, and some centers routinely admit all patients to an ICU after cesarean hysterectomy for intensive hemodynamic, cardiorespiratory, and hematologic monitoring. Intensive care may be indicated for ventilator support in women who require massive fluid and transfusion resuscitation. Given the frequency of transfusion, some PAS patients may have transfusion-related acute lung injury or acute pulmonary edema. In the case of disseminated intravascular coagulation (DIC), pelvic packing and delayed closure of the abdomen may be useful. In these patients, ICU care is appropriate.

Several other types of expertise are also useful for PAS CoE. These include surgeons with expertise in pelvic and urologic surgery and may include general obstetrician gynecologists, maternal fetal medicine specialists, gynecologic oncologists, and urologists. Rarely, additional expertise in trauma, vascular, or gastrointestinal surgery may be required. Surgical support staff who are familiar with the equipment and instruments needed for safe and rapid hysterectomies are also necessary for optimal surgical outcomes. Since preterm birth often occurs, neonatologists 


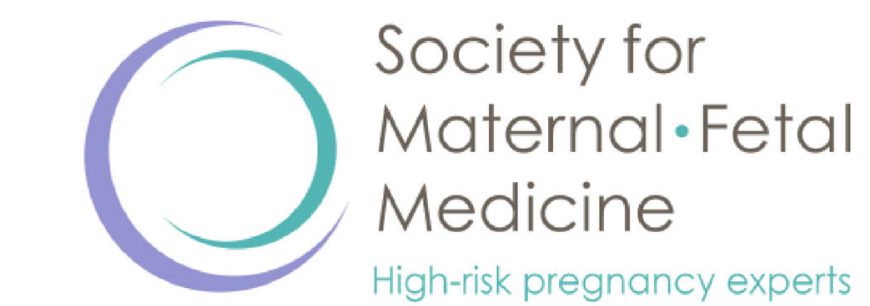

\section{SMFM Checklist for Unexpected Morbidly Adherent Placenta}

Intended for use when morbidly adherent placenta is first encountered at the time of labor onset or delivery, and was not diagnosed antenatally.

\section{Diagnosis Before Delivery (e.g. bleeding prior to delivery):}

If located at facility without accreta experience:

Assess stability (vital signs, extent of blood loss, fetal monitoring status)

$\square$ Assess and prepare surgical help, equipment, \& transfusion capability (see contact numbers below)

$\square$ Consider transport to facility with accreta experience if patient is stable

$\square$ Contact possible accepting facility

\section{Diagnosis at Laparotomy:}

If located at facility without accreta experience and if transport may be option:

Assess stability (vital signs, extent of blood loss, hemodynamics, fetal status)

Assess placental location visually and by intra-operative ultrasound

$\square$ Assess and prepare resources (surgical help, equipment, \& transfusion capability; see contact numbers below)

$\square$ Assess transport capabilities (includes contact to possible accepting facility)

$\square$ Consider delaying uterine incision until resources available at facility (if maternal and fetal status permits), or

$\square$ Consider no uterine incision, close abdomen, \& prepare for transport to referral center (if fetal and maternal status permits), or

$\square$ Consider delivery of fetus by fundal incision (or incision that avoids placenta if mapping is possible), closure of uterus and abdomen, \& transport if stable and appropriate

If transporting, photograph intraoperative findings for receiving facility

Fig. 1 One page of the SMFM Checklist for unexpected cases of PAS 
and a neonatal intensive care unit are also crucial. Finally, it is important to have skilled mental health care workers to provide emotional support to women and families with PAS. These individuals are at increased risk for depression, anxiety, and PTSD and often have complex emotions about having a hysterectomy.

\section{Operationalizing Multidisciplinary Accreta Care}

To operationalize a MDT approach, we recommend the following:

First, centers should implement an on-call PAS MDT. Up to $50 \%$ of patients with PAS will undergo unscheduled preterm delivery, even when delivery is planned for 34 35 -week gestation [3]. These deliveries are often more difficult and morbid than planned cases and should not be left to less-experience operators just because they are unscheduled.

Second, the PAS MDT should meet regularly to discuss cases at patient care conferences. These conferences may resemble multidisciplinary tumor board conferences in oncology, where upcoming cases are planned and past cases are reviewed. Continuous quality improvement and system optimization happens at these conferences and has exponential downstream improvement benefits. For each patient, care is improved because her case is optimally planned, prepared, and timed. Unique medical conditions (e.g., comorbid conditions, extensive past surgical history) can be discussed. Also, unique social circumstances (e.g., non-proximity to the hospital, social chaos) can be addressed.

Third, a standard protocol for management of PAS should be established at each center. Though too few quality data are available to inform a single evidence-based standard of care for management of PAS, a standardized approach to diagnosis, antenatal management, delivery timing, anesthesia, transfusion preparedness, and operative management is key to reducing errors and confusion. Preoperative checklists and team-training exercises can enhance this process and improve interdisciplinary care processes $[14,15]$.

Fourth, lower-acuity obstetric practices and centers should prepare for the inevitability of managing patients with PAS. Clear indications for antenatal referral to higher levels of maternal care should be protocolized and encouraged. For example, a practice with more limited imaging capabilities may decide to refer all patients with clinical or sonographic risk factors for accreta to a referral center for diagnosis or to rule out of PAS. Similarly, a hospital with no capability for massive transfusion or no 24-h interventional radiology should refer patients with any risk of PAS to centers with these resources. Finally, even loweracuity hospitals should have a written plan and transfer protocols in place for when a case of PAS is diagnosed during delivery.

\section{Checklists}

To improve readiness, preparation, and response to cases of PAS at obstetric hospitals, the Society for Maternal-Fetal Medicine developed two helpful checklists (found at https:// www.smfm.org/checklists-and-safety-bundles). These checklists incorporate the fundamentals already discussed in this review.

The first is a checklist for cases of suspected PAS. It is designed as a tool to be adapted in centers that plan surgical cases for PAS. It includes sections on antenatal management, operating room planning, MDT coordination, blood product planning, the surgical approach, and postoperative planning. One section allows centers caring for PAS to list out local contact numbers (e.g., trauma surgeon on call number).

The second is a checklist for unexpected cases of PAS and is designed for institutions that may be under-resourced to manage PAS routinely but who will nevertheless encounter PAS in practice (Fig. 1). Subsections address circumstances in which a diagnosis of PAS is made in the third trimester due to bleeding in patients who are stable enough for transport. Another section focuses on what to do in the event of diagnosis at the time of laparotomy. A section for emergency contact numbers to be filled in locally is also available for this checklist.

\section{Conclusions}

The key to success and safe treatment of patients with PAS is in the implementation of a coordinated MDT of highly skilled and experienced providers within a system having ample subspecialty resources. This review has addressed evidence and guidelines supporting a benefit to PAS management by a multidisciplinary care team. We recommend referral to tertiary care center with experience in the diagnosis and treatment of PAS, or an Accreta CoE, when women have clinical or sonographic risk for PAS (e.g., placenta previa in the setting of prior cesarean delivery). MDT coordination is an absolute requirement in patients with PAS. 


\section{Compliance with Ethics Guidelines}

Conflict of Interest Brett D. Einerson and Robert M. Silver declare no conflict of interest.

Human and Animal Rights and Informed Consent This article does not contain any studies with human or animal subjects performed by any of the authors.

\section{References}

Papers of particular interest, published recently, have been highlighted as:

- Of importance

-• Of major importance

1. Wright JD, Herzog TJ, Shah M, Bonanno C, Lewin SN, Cleary K, et al. Regionalization of care for obstetric hemorrhage and its effect on maternal mortality. Obstet Gynecol. 2010;115:1194-200 In a large nationally-representative sample, the authors convincingly demonstrate a difference in maternal mortality between high- and low-volume hospitals for women undergoing peripartum hysterectomy, $35 \%$ of whom had a diagnosis of placenta accreta spectrum.

2. Eller AG, Bennett MA, Sharshiner M, Masheter C, Soisson AP, Dodson $\mathrm{M}$, et al. Maternal morbidity in cases of placenta accreta managed by a multidisciplinary care team compared with standard obstetric care. Obstet Gynecol. 2011;117:331-7 This state-wide evaluation of placenta accreta management was the first and still largest study to show reduced maternal morbidity for women with PAS receiving care within multidisciplinary care setting.

3. Shamshirsaz AA, Fox KA, Salmanian B, Diaz-Arrastia CR, Lee W, Baker BW, et al. Maternal morbidity in patients with morbidly adherent placenta treated with and without a standardized multidisciplinary approach. Am J Obstet Gynecol. 2015;212:218.e1-9.

4. Shamshirsaz AA, Fox KA, Erfani H, Clark SL, Salmanian $\mathrm{B}$, Baker BW, et al. Multidisciplinary team learning in the management of the morbidly adherent placenta: outcome improvements over time. Am J Obstet Gynecol. 2017;216: 612.e1-5.
5. Placenta accreta spectrum. Obstetric care consensus no. 7. American College of Obstetricians and Gynecologists. Obstet Gynecol. 2018;132:e259-75.

6. Levels of maternal care. Obstetric care consensus no. 2. American College of Obstetricians and Gynecologists. Obstet Gynecol. 2015;125:502-15.

7. Jauniaux ERM, Alfirevic Z, Bhide AG, Belfort MA, Burton GJ, Collins SL, Dornan S, Jurkovic D, Kayem G, Kingdom J, Silver R, Sentilhes L on behalf of the Royal College of Obstetricians and Gynaecologists. Placenta Praevia and Placenta Accreta: Diagnosis and Management. Green-top Guideline No. 27a. BJOG 2018

8. Wright JD, Silver RM, Bonanno C, Gaddipati S, Lu YS, Simpson LL, et al. Practice patterns and knowledge of obstetricians and gynecologists regarding placenta accreta. $\mathrm{J}$ Matern Fetal Neonatal Med. 2013;26:1602-9.

9. Fitzpatrick KE, Sellers S, Spark P, Kurinczuk JJ, Brocklehurst P, Knight M. The management and outcomes of placenta accreta, increta, and percreta in the UK: a population-based descriptive study. BJOG. 2014;121:62-71.

10. Bailit JL, Grobman WA, Rice MM, Reddy UM, Wapner RJ, Varner MW, et al. Morbidly adherent placenta treatments and outcomes. Obstet Gynecol. 2015;125:683-9.

11. Eller AG, Porter TF, Soisson P, Silver RM. Optimal management strategies for placenta accreta. BJOG. 2009;116:648-54.

12. Warshak CR, Ramos GA, Eskander R, Benirschke K, Saenz CC, Kelly TF, et al. Effect of predelivery diagnosis in 99 consecutive cases of placenta accreta. Obstet Gynecol. 2010;115:65-9.

13.• Silver, RM FKA, Barton JR, Abuhamad AZ, Simhan H, Huls CK, et al. Center of excellence for placenta accreta. Am J Obstet Gynecol. 2015;212:561-8 This position statement from a group of experts at experienced accreta referral centers first defined the concept and characteristics of a multidisciplinary accreta team.

14. Skupski DW, Brady D, Lowenwirt IP, Sample J, Lin SN, Lohana R, et al. Improvement in outcomes of major obstetric hemorrhage through systematic change. Obstet Gynecol. 2017;130(4):770-7.

15. Fransen AF, van de Ven J, Schuit E, van Tetering A, Mol BW, Oei SG. Simulation-based team training for multi-professional obstetric care teams to improve patient outcome: a multicentre, cluster randomised controlled trial. BJOG. 2017;124(4):641-50.

Publisher's Note Springer Nature remains neutral with regard to jurisdictional claims in published maps and institutional affiliations. 